Article

\title{
Exact Time-Dependent Queue-Length Solution to a Discrete-Time Geo/D/1 Queue
}

\author{
Jung Woo Baek ${ }^{1}$ (D) and Yun Han Bae ${ }^{2, *}$ \\ 1 Department of Industrial Engineering, Chosun University, Gwangju 61452, Korea \\ 2 Department of Mathematics Education, Sangmyung University, Seoul 03016, Korea \\ * Correspondence: yhbae@smu.ac.kr
}

Received: 18 July 2019; Accepted: 6 August 2019; Published: 7 August 2019

\begin{abstract}
Time-dependent solutions to queuing models are beneficial for evaluating the performance of real-world systems such as communication, transportation, and service systems. However, restricted results have been reported due to mathematical complexity. In this study, we present a time-dependent queue-length formula for a discrete-time Geo / D / 1 queue starting with a positive number of initial customers. We derive the time-dependent formula in closed form.
\end{abstract}

Keywords: time-dependent queue-length probability; discrete-time Geo / D /1 queue; closed-form solution

\section{Introduction}

Queuing models have been widely used for performance evaluation of practical systems such as communication, transportation, and service systems. For the most part, the previous studies have addressed stationary solutions of queuing models for the optimal control of long-term system performance. However, time-dependent solutions to queuing systems are more meaningful for practical systems; a good example was presented in Griffiths et al. [1], where the authors applied the transient solution of an $M / E_{k} / 1$ queue to model and analyze a 24-hour traffic profile on the Severn Bridge in England.

The pioneering results on the transient results were presented by Luchak [2] and Saaty [3]. Using a modified Bessel function, they presented the transient queue-length distribution of an $M / M / 1$ queue starting with a positive number of initial customers. Later, computationally efficient solutions were presented in many other studies [4-8]. For $M / E_{k} / 1$ queues, several results have been presented. Griffiths et al. [1,9] and Leonenko [10] derived transient solutions for $M / E_{k} / 1$ queues starting with a positive number of initial customers, and Baek et al. [11] extended their results to be applied to analysis in a single busy period. Furthermore, Kapodistria et al. [12] presented the time-dependent solutions to a linear birth/immigration-death process with binomial catastrophes. They presented general results that include a background random environment.

The first study on queues with deterministic service times was conducted by Garcia et al. [13]. Using the matrix-analytic method, they derived the transient queue-length distribution of an $M / D / 1 / N$ queue in a computationally efficient form. Later, the queue-length formula was extended to an $M / D / c$ queue by Franx [14]. Recently, extensive results on the transient solution of the $M / D / 1$ queue were presented by Baek et al. [15]. They derived not only the queue-length probability but also the waiting time distribution in closed form.

The aforementioned studies focused only on the queues in a continuous-time domain. Time-dependent solutions to queues in a discrete-time domain are even scarcer. Parthasarathy and Sudhesh [16] were the first to derive the transient queue-length solution to a discrete-time Geo / Geo/1 queue. Employing a generating function and continued fractions, they obtained a computationally efficient solution. Kim [17] studied the same model and presented the solution in a formal form. 
However, we failed to find any result related to the time-dependent solution to the discrete-time queue with a constant service time. From this perspective, we present a time-dependent queue-length solution to a Geo/D/1 queue. To the best of our knowledge, our proposed results have not been noted in existing literature.

\section{Main Results}

In this section, we present the main results. Consider a discrete-time Geo/D/1 queuing system with an infinite buffer in which the timeline is divided into intervals of equal length called time slots. Customers arrive into the system according to the Bernoulli process at a rate of $p$, and the service times are assumed to be a constant of $D$. We assume that arrivals and service completion can occur only at the slot boundaries. More specifically, we consider the late-arrival model [18]. Therefore, we assume that customer arrivals can occur only in $(n-, n)$ and services can be completed only in $(n, n+)$.

Let $N_{0}$ be the number of new initial customers at time slot 0 . We define $N(n)$ as the number of customers at the end of the time slot $n$ and with the following probability:

$$
Q_{k}^{(j)}(n)=\operatorname{Pr}\left[N(n)=k \mid N_{0}=j\right], \quad(k \geq 0, j \geq 0),(n>0)
$$

Because $Q_{0}^{(j)}(n)$ plays a major role in deriving $Q_{k}^{(j)}(n)$, we first derive the probability $Q_{0}^{(j)}(n)$ in the following theorem.

\section{Theorem 1. We have}

$$
Q_{0}^{(j)}(n)=\sum_{r=0}^{\left\lfloor\frac{n}{D}\right\rfloor-j}\left(\begin{array}{l}
n \\
r
\end{array}\right) p^{r}(1-p)^{n-r}\left(1-\frac{r}{n} D\right)
$$

Proof. Let us define $A(n)$ as the number of arrivals during the time interval $(0, n]$ and $\tau_{i(r)}(n)$ as the $i$-th arrival epoch under the condition that $A(n)=r$. We note that the service time is a constant $D$ and the system starts with $j$ initial customers. Therefore, conditioning on $A(n)$, we have

$$
\begin{aligned}
& Q_{0}^{(j)}(n)= \sum_{r=0}^{\infty} \operatorname{Pr}\left(\tau_{1(r)}(n)<\tau_{2(r)}(n)<\cdots<\tau_{r(r)}(n)\right. \\
&\left.\tau_{1(r)}(n) \leq n-r D, \tau_{2(r)}(n) \leq n-(r-1) D, \ldots, \tau_{r(r)}(n) \leq n-D \mid A(n)=r\right) \\
& \quad \times \operatorname{Pr}(A(n)=r) U(n \geq(j+r) D)
\end{aligned}
$$

where $U(A)$ is an indicator function which takes 1 , if $\mathrm{A}$ is true, or 0 , and the second equality holds because it is obvious that $\tau_{1(r)}(n)<\tau_{2(r)}(n)<\cdots<\tau_{r(r)}(n)$.

Equation (2) means that the system can be empty at time slot $n$ only when the $i$-th customer among $r$ customers arrives before $[n-(r-(i-1)) D]$-th time slot $(1 \leq i \leq r)$ under the condition that $A(n)=r$. Since the arrival process is Bernoulli process with rate $p$, we have $\operatorname{Pr}(A(n)=r)=$ $\left(\begin{array}{l}n \\ r\end{array}\right) p^{r}(1-p)^{n-r}$. Next, to complete Equation (2), we need to derive $\operatorname{Pr}\left(\tau_{1(r)}(n) \leq n-r D, \tau_{2(r)}(n) \leq\right.$ $\left.n-(r-1) D, \ldots, \tau_{r(r)}(n) \leq n-D \mid A(n)=r\right)$. For $1 \leq n_{1}<n_{2}<\cdots<n_{r} \leq n$, it is not difficult to have

$$
\begin{aligned}
& \operatorname{Pr}\left(\tau_{1(r)}(n)=n_{1}, \tau_{2(r)}(n)=n_{2}, \cdots, \tau_{r(r)}(n)=n_{r} \mid A(n)=r\right) \\
& =\frac{\operatorname{Pr}\left(\tau_{1(r)}(n)=n_{1}, \tau_{2(r)}(n)=n_{2}, \cdots, \tau_{r(r)}(n)=n_{r}, A(n)=r\right)}{\operatorname{Pr}(A(n)=r)}=\frac{p^{r}(1-p)^{n-r}}{\left(\begin{array}{c}
n \\
r
\end{array}\right) p^{r}(1-p)^{n-r}}=\frac{1}{\left(\begin{array}{c}
n \\
r
\end{array}\right)} .
\end{aligned}
$$


Therefore, we have

$$
\begin{aligned}
& \operatorname{Pr}\left(\tau_{1(r)}(n) \leq n-r D, \tau_{2(r)}(n) \leq n-(r-1) D, \ldots, \tau_{r(r)}(n) \leq n-D \mid A(n)=r\right) \\
& =\sum_{n_{1}=1}^{n-r D} \sum_{n_{2}=n_{1}+1}^{n-(r-1) D} \cdots \sum_{n_{r}=n_{r-1}+1}^{n-D} \operatorname{Pr}\left(\tau_{1(r)}(n)=n_{1}, \tau_{2(r)}(n)=n_{2}, \cdots, \tau_{r(r)}(n)=n_{r} \mid A(n)=r\right) \\
& =\sum_{n_{1}=1}^{n-r D} \sum_{n_{2}=n_{1}+1}^{n-(r-1) D} \cdots \sum_{n_{r}=n_{r-1}+1}^{n-D} \frac{1}{\left(\begin{array}{c}
n \\
r
\end{array}\right)} .
\end{aligned}
$$

Using Equation (4) in Equation (2), we obtain

$$
Q_{0}^{(j)}(n)=\sum_{r=0}^{\left\lfloor\frac{n}{D}\right\rfloor-j}\left[\sum_{n_{1}=1}^{n-r D} \sum_{n_{2}=n_{1}+1}^{n-(r-1) D} \cdots \sum_{n_{r}=n_{r-1}+1}^{n-D} \frac{1}{\left(\begin{array}{c}
n \\
r
\end{array}\right)}\right]\left(\begin{array}{l}
n \\
r
\end{array}\right) p^{r}(1-p)^{n-r}
$$

Now, to obtain Equation (1), it is sufficient to prove that

$$
\sum_{n_{1}=1}^{n-r D} \sum_{n_{2}=n_{1}+1}^{n-(r-1) D} \cdots \sum_{n_{r}=n_{r-1}+1}^{n-D} \frac{1}{\left(\begin{array}{c}
n \\
r
\end{array}\right)}=1-\frac{r}{n} D
$$

We use mathematical induction for the proof of Equation (6). For $r=1$, we trivially have $\sum_{n_{1}=1}^{n-D} \frac{1}{\left(\begin{array}{c}n \\ 1\end{array}\right)}=1-\frac{1}{n} D$. We now assume that Equation (6) holds for $r=l$. Then, for $r=l+1$, we have

$$
\begin{aligned}
& \sum_{n_{1}=1}^{n-(l+1) D} \sum_{n_{2}=n_{1}+1}^{n-l D} \cdots \sum_{n_{l+1}=n_{l}+1}^{n-D} \frac{1}{\left(\begin{array}{c}
n \\
l+1
\end{array}\right)} \\
= & \sum_{n_{1}=1}^{n-(l+1) D}\left[\sum_{i_{1}=1}^{n-n_{1}-l D} \cdots \sum_{i_{l}=i_{l-1}+1}^{n-n_{1} D} \frac{1}{\left(\begin{array}{c}
n-n_{1} \\
l
\end{array}\right)}\right] \frac{\left(\begin{array}{c}
n-n_{1} \\
l
\end{array}\right)}{\left(\begin{array}{c}
n \\
l+1
\end{array}\right)}=\sum_{n_{1}=1}^{n-(l+1) D}\left[1-\frac{l D}{n-n_{1}}\right] \frac{\left(\begin{array}{c}
n-n_{1} \\
l
\end{array}\right)}{\left(\begin{array}{c}
n \\
l+1
\end{array}\right)},
\end{aligned}
$$

where we use $i_{k}=n_{k+1}-n_{1}$ for $k \geq 1$.

We now have

$$
\begin{aligned}
& \sum_{n_{1}=1}^{n-(l+1) D}\left[1-\frac{l D}{n-n_{1}}\right] \frac{\left(\begin{array}{c}
n-n_{1} \\
l
\end{array}\right)}{\left(\begin{array}{c}
n \\
l+1
\end{array}\right)}=\sum_{n_{1}=1}^{n-(l+1) D} \frac{n-n_{1}-l D}{n-n_{1}} \cdot \frac{\left(n-n_{1}\right) ! \cdot(n-l-1) !}{n ! \cdot\left(n-n_{1}-l\right) !} \cdot(l+1) \\
= & \frac{n-1-l D}{n-1} \cdot \frac{(n-1) ! \cdot(n-l-1) !}{n ! \cdot(n-1-l) !} \cdot(l+1)+\frac{n-2-l D}{n-2} \cdot \frac{(n-2) ! \cdot(n-l-1) !}{n ! \cdot(n-2-l) !} \cdot(l+1) \\
& \quad+\cdots+\frac{(l+1) D-l D}{(l+1) D} \cdot \frac{((l+1) D) ! \cdot(n-l-1) !}{n ! \cdot((l+1) D-l) !} \cdot(l+1) \\
= & \sum_{m=(l+1) D}^{n-1} \frac{m-l D}{m} \cdot \frac{m ! \cdot(n-l-1) !}{n ! \cdot(m-l) !} \cdot(l+1) \\
= & \left(1-\frac{(l+1) D}{n}\right) \cdot \frac{(n-l-1) !}{(n-1) !} \cdot \frac{l+1}{n-(l+1) D} \sum_{m=(l+1) D}^{n-1} \frac{(m-l D) \cdot(m-1) !}{(m-l) !} .
\end{aligned}
$$


In Equation (8), we have

$$
\begin{aligned}
& \sum_{m=(l+1) D}^{n-1} \frac{(m-l D) \cdot(m-1) !}{(m-l) !} \\
= & \sum_{m=0}^{n-1}\left[\frac{m !}{(m-l) !}-l D \frac{(m-1) !}{(m-l) !}\right]-\sum_{m=0}^{(l+1) D-1}\left[\frac{m !}{(m-l) !}-l D \frac{(m-1) !}{(m-l) !}\right] \\
= & l !\left[\sum_{m=0}^{n-1}\left(\begin{array}{c}
m \\
l
\end{array}\right)-D \cdot \sum_{m=0}^{n-1}\left(\begin{array}{c}
m-1 \\
l-1
\end{array}\right)-\sum_{m=0}^{(l+1) D-1}\left(\begin{array}{c}
m \\
l
\end{array}\right)+D \cdot \sum_{m=0}^{(l+1) D-1}\left(\begin{array}{c}
m-1 \\
l-1
\end{array}\right)\right] \\
= & l !\left[\left(\begin{array}{c}
n \\
l+1
\end{array}\right)-D \cdot\left(\begin{array}{c}
n-1 \\
l
\end{array}\right)-\left(\begin{array}{c}
(l+1) D \\
l+1
\end{array}\right)+D \cdot\left(\begin{array}{c}
(l+1) D-1 \\
l
\end{array}\right)\right] \\
= & l ! \cdot\left(\begin{array}{c}
n \\
l+1
\end{array}\right)-l ! \cdot D \cdot\left(\begin{array}{c}
n-1 \\
l
\end{array}\right) \\
= & \frac{\left(\begin{array}{l}
n-1) ! \\
(n-l-1) !
\end{array} \cdot \frac{n-(l+1) D}{l+1},\right.}{l}
\end{aligned}
$$

where we use $\sum_{j=0}^{n}\left(\begin{array}{c}j \\ m\end{array}\right)=\left(\begin{array}{c}n+1 \\ m+1\end{array}\right)$ for the third equality.

Using Equations (8) and (9) in Equation (7), we obtain

$$
\sum_{n_{1}=1}^{n-(l+1) D} \sum_{n_{2}=n_{1}+1}^{n-l D} \cdots \sum_{n_{l+1}=n_{l}+1}^{n-D} \frac{1}{\left(\begin{array}{c}
n \\
l+1
\end{array}\right)}=1-\frac{l+1}{n} D .
$$

Therefore, we prove that Equation (6) holds for $r=1,2,3, \cdots$.

Next, we derive $Q_{k}^{(j)}(n),(k \geq 1)$. We have the following theorem.

Theorem 2. We have

$$
\begin{aligned}
Q_{k}^{(j)}(n)= & \left(\begin{array}{c}
n \\
k+\left\lfloor\frac{n}{D}\right\rfloor-j
\end{array}\right) p^{k+\left\lfloor\frac{n}{D}\right\rfloor-j}(1-p)^{n-\left(k+\left\lfloor\frac{n}{D}\right\rfloor-j\right)} \\
& \left.+\sum_{r=0}^{\left\lfloor\frac{n}{D}\right\rfloor-j-1\left\lfloor\frac{n}{D}\right\rfloor-j-1-r} \sum_{m=0}^{n-(r+1) D} \begin{array}{c}
n \\
m
\end{array}\right) p^{m}(1-p)^{n-(r+1) D-m} \\
& \times\left(\frac{(k+r+1)-p-p[(r+1) D]}{(1-p)(k+r+1)}\right)\left(1-\frac{m}{\frac{n}{D}-r-1}\right)\left(\begin{array}{c}
(r+1) D \\
k+r
\end{array}\right) p^{k+r}(1-p)^{(r+1) D-(k+r) .}
\end{aligned}
$$

Proof. Let us define $A^{\text {last }}$ and $\tau^{\text {last }}$ as the number of arrivals during the last busy period and the time slot at which the last busy period starts, respectively. We then have

$$
\begin{aligned}
Q_{k}^{(j)}(n)= & \operatorname{Pr}\left[N(n)=k \mid N_{0}=j\right] \\
= & \operatorname{Pr}\left[N(n)=k, 0 \leq \tau^{\text {last }} \leq n-\left(\left\lfloor\frac{n}{D}\right\rfloor-j\right) D \mid N_{0}=j\right] \\
& +\sum_{r=0}^{\left\lfloor\frac{n}{D}\right\rfloor-j-1} \operatorname{Pr}\left[N(n)=k, n-(r+1) D<\tau^{\text {last }} \leq n-r D \mid N_{0}=j\right] .
\end{aligned}
$$

To complete Equation (11), we first derive the first term in the second equality. We again note that the system starts with $j$ customers, and the service time is a constant $D$. Therefore, if the last busy period starts before the $n-\left(\left\lfloor\frac{n}{D}\right\rfloor-j\right) D$-th time slot, $\left\lfloor\frac{n}{D}\right\rfloor-j$ customers among newly arrived 
customers should be served during the last busy period. Then, to become $N(n)=k$ under this situation, $k+\left\lfloor\frac{n}{D}\right\rfloor-j$ customers should arrive in the system, and we have

$$
\begin{aligned}
\operatorname{Pr} & {\left[N(n)=k, 0 \leq \tau^{\text {last }} \leq n-\left(\left\lfloor\frac{n}{D}\right\rfloor-j\right) D \mid N_{0}=j\right] } \\
& =\operatorname{Pr}\left[N(n)=k, 0 \leq \tau^{\text {last }} \leq n-\left(\left\lfloor\frac{n}{D}\right\rfloor-j\right) D, A^{\text {last }}=k+\left\lfloor\frac{n}{D}\right\rfloor-j \mid N_{0}=j\right] .
\end{aligned}
$$

Next, we have

$$
\begin{aligned}
\operatorname{Pr}\left[N(n)=k, 0 \leq \tau^{\text {last }} \leq n-\left(\left\lfloor\frac{n}{D}\right\rfloor-j\right) D, A^{\text {last }}=k+\left\lfloor\frac{n}{D}\right\rfloor-j \mid N_{0}=j\right] \\
=\operatorname{Pr}\left[N(n)=k, 0 \leq \tau^{\text {last }} \leq n, A^{\text {last }}=k+\left\lfloor\frac{n}{D}\right\rfloor-j \mid N_{0}=j\right] \\
\quad-\operatorname{Pr}\left[N(n)=k, n-\left(\left\lfloor\frac{n}{D}\right\rfloor-j\right) D<\tau^{\text {last }} \leq n, A^{\text {last }}=k+\left\lfloor\frac{n}{D}\right\rfloor-j \mid N_{0}=j\right] \\
=\operatorname{Pr}\left[k \leq N(n) \leq k+\left\lfloor\frac{n}{D}\right\rfloor-j, 0 \leq \tau^{\text {last }} \leq n, A^{\text {last }}=k+\left\lfloor\frac{n}{D}\right\rfloor-j \mid N_{0}=j\right] \\
\quad-\operatorname{Pr}\left[k+1 \leq N(n) \leq k+\left\lfloor\frac{n}{D}\right\rfloor-j, 0 \leq \tau^{\text {last }} \leq n, A^{\text {last }}=k+\left\lfloor\frac{n}{D}\right\rfloor-j \mid N_{0}=j\right] \\
\quad-\operatorname{Pr}\left[k \leq N(n) \leq k+\left\lfloor\frac{n}{D}\right\rfloor-j, n-\left(\left\lfloor\frac{n}{D}\right\rfloor-j\right) D<\tau^{\text {last }} \leq n, A^{\text {last }}=k+\left\lfloor\frac{n}{D}\right\rfloor-j \mid N_{0}=j\right] \\
\quad+\operatorname{Pr}\left[k+1 \leq N(n) \leq k+\left\lfloor\frac{n}{D}\right\rfloor-j, n-\left(\left\lfloor\frac{n}{D}\right\rfloor-j\right) D<\tau^{\text {last }} \leq n, A^{\text {last }}=k+\left\lfloor\frac{n}{D}\right\rfloor-j \mid N_{0}=j\right] \\
=\operatorname{Pr}\left[k \leq N(n) \leq k+\left\lfloor\frac{n}{D}\right\rfloor-j, 0 \leq \tau^{\text {last }} \leq n, A^{\text {last }}=k+\left\lfloor\frac{n}{D}\right\rfloor-j \mid N_{0}=j\right] \\
\quad-\operatorname{Pr}\left[k \leq N(n) \leq k+\left\lfloor\frac{n}{D}\right\rfloor-j, n-\left(\left\lfloor\frac{n}{D}\right\rfloor-j\right) D<\tau^{\text {last }} \leq n, A^{\text {last }}=k+\left\lfloor\frac{n}{D}\right\rfloor-j \mid N_{0}=j\right] \\
\quad-\operatorname{Pr}\left[k+1 \leq N(n) \leq k+\left\lfloor\frac{n}{D}\right\rfloor-j, 0 \leq \tau^{\text {last }} \leq n-\left(\left\lfloor\frac{n}{D}\right\rfloor-j\right) D, A^{\text {last }}=k+\left\lfloor\frac{n}{D}\right\rfloor-j \mid N_{0}=j\right] .
\end{aligned}
$$

In Equation (13), we have

$$
\operatorname{Pr}\left[k+1 \leq N(n) \leq k+\left\lfloor\frac{n}{D}\right\rfloor-j, 0 \leq \tau^{\text {last }} \leq n-\left(\left\lfloor\frac{n}{D}\right\rfloor-j\right) D, A^{\text {last }}=k+\left\lfloor\frac{n}{D}\right\rfloor-j \mid N_{0}=j\right]=0
$$

because $\left\lfloor\frac{n}{D}\right\rfloor-j$ customers among the newly arrived customers should be served during the last busy period, if the busy period starts in the time interval $\left[0, n-\left(\left\lfloor\frac{n}{D}\right\rfloor-j\right) D\right]$.

Furthermore, we note that

$$
\operatorname{Pr}\left[N(n)=k, n-\left(\left\lfloor\frac{n}{D}\right\rfloor-j\right) D<\tau^{\text {last }} \leq n, A^{\text {last }}=k+\left\lfloor\frac{n}{D}\right\rfloor-j \mid N_{0}=j\right]=0 .
$$

We then have

$$
\begin{gathered}
\operatorname{Pr}\left[N(n)=k, 0 \leq \tau^{\text {last }} \leq n-\left(\left\lfloor\frac{n}{D}\right\rfloor-j\right) D, A^{\text {last }}=k+\left\lfloor\frac{n}{D}\right\rfloor-j \mid N_{0}=j\right] \\
=\operatorname{Pr}\left[k \leq N(n) \leq k+\left\lfloor\frac{n}{D}\right\rfloor-j, 0 \leq \tau^{\text {last }} \leq n, A^{\text {last }}=k+\left\lfloor\frac{n}{D}\right\rfloor-j \mid N_{0}=j\right] \\
\quad-\operatorname{Pr}\left[k+1 \leq N(n) \leq k+\left\lfloor\frac{n}{D}\right\rfloor-j, n-\left(\left\lfloor\frac{n}{D}\right\rfloor-j\right) D<\tau^{\text {last }} \leq n, A^{\text {last }}=k+\left\lfloor\frac{n}{D}\right\rfloor-j \mid N_{0}=j\right] \\
=\left(\begin{array}{c}
n \\
k+\left\lfloor\frac{n}{D}\right\rfloor-j
\end{array}\right) \cdot p^{k+\left\lfloor\frac{n}{D}\right\rfloor-j} \cdot(1-p)^{n-\left(k+\left\lfloor\frac{n}{D}\right\rfloor-j\right)} \\
\quad-Q_{0}^{(j)}\left[n-\left(\left\lfloor\frac{n}{D}\right\rfloor-j\right) D\right] \cdot\left(\begin{array}{c}
\left(\left\lfloor\frac{n}{D}\right\rfloor-j\right) D \\
k+\left\lfloor\frac{n}{D}\right\rfloor-j
\end{array}\right) \cdot p^{k+\left\lfloor\frac{n}{D}\right\rfloor-j} \cdot(1-p)^{\left(\left\lfloor\frac{n}{D}\right\rfloor-j\right) D-\left(k+\left\lfloor\frac{n}{D}\right\rfloor-j\right)} .
\end{gathered}
$$


Next, we need to derive $\operatorname{Pr}\left[N(n)=k, n-(r+1) D<\tau^{\text {last }} \leq n-r D \mid N_{0}=j\right], \quad(0 \leq r \leq$ $\left.\left\lfloor\frac{n}{D}\right\rfloor-j-1\right)$ in Equation (11). When $r=0$, it is not difficult to have

$$
\operatorname{Pr}\left[N(n)=k, n-D<\tau^{\text {last }} \leq n \mid N_{0}=j\right]=Q_{0}^{(j)}(n-D)\left[\left(\begin{array}{l}
D \\
k
\end{array}\right) \cdot p^{k} \cdot(1-p)^{D-k}\right]
$$

because no service can be completed during the time interval $(n-D, n]$.

In Equation (17), we assume that $\left(\begin{array}{l}D \\ k\end{array}\right)=0$, if $k>D$. Next, we consider the case with $1 \leq r \leq$ $\left\lfloor\frac{n}{D}\right\rfloor-j-1$. Applying a similar approach used in Equations (12)-(16), we have

$$
\begin{aligned}
& \operatorname{Pr}\left[N(n)=k, n-(r+1) D<\tau^{\text {last }} \leq n-r D \mid N_{0}=j\right] \\
& =\operatorname{Pr}\left[N(n)=k, n-(r+1) D<\tau^{\text {last }} \leq n-r D, A^{\text {last }}=k+r \mid N_{0}=j\right] \\
& =\operatorname{Pr}\left[k \leq N(n) \leq k+r, n-(r+1) D<\tau^{\text {last }} \leq n, A^{\text {last }}=k+r \mid N_{0}=j\right] \\
& \quad-\operatorname{Pr}\left[k+1 \leq N(n) \leq k+r, n-r D<\tau^{\text {last }} \leq n, A^{\text {last }}=k+r \mid N_{0}=j\right] \\
& =Q_{0}^{(j)}(n-(r+1) D) \cdot\left(\begin{array}{c}
(r+1) D \\
k+r
\end{array}\right) \cdot p^{k+r} \cdot(1-p)^{(r+1) D-(k+r)} \\
& \quad-Q_{0}^{(j)}(n-r D) \cdot\left(\begin{array}{c}
r D \\
k+r
\end{array}\right) \cdot p^{k+r} \cdot(1-p)^{r D-(k+r)}, \quad\left(1 \leq r \leq\left\lfloor\frac{n}{D}\right\rfloor-j-1\right) .
\end{aligned}
$$

Using Equations (12) and (16)-(18) in Equation (11), we can obtain the simplified form as

$$
\begin{aligned}
Q_{k}^{(j)}(n)= & \left(\begin{array}{c}
n \\
k+\left\lfloor\frac{n}{D}\right\rfloor-j
\end{array}\right) \cdot p^{k+\left\lfloor\frac{n}{D}\right\rfloor-j} \cdot(1-p)^{n-\left(k+\left\lfloor\frac{n}{D}\right\rfloor-j\right)} \\
& +\sum_{r=0}^{\left\lfloor\frac{n}{D}\right\rfloor-j-1} Q_{0}^{(j)}(n-(r+1) D) \cdot\left(\begin{array}{c}
(r+1) D \\
k+r
\end{array}\right) \cdot p^{k+r} \cdot(1-p)^{(r+1) D-(k+r)} \\
& -\sum_{r=0}^{\left\lfloor\frac{n}{D}\right\rfloor-j-1} Q_{0}^{(j)}(n-(r+1) D) \cdot\left(\begin{array}{c}
(r+1) D \\
k+r+1
\end{array}\right) \cdot p^{k+r+1} \cdot(1-p)^{(r+1) D-(k+r+1)}
\end{aligned}
$$

From Equation (1), we have

$$
Q_{0}^{(j)}[(n-(r+1) D)]=\sum_{m=0}^{\left\lfloor\frac{n}{D}\right\rfloor-(r+1)-j}\left(\begin{array}{c}
n-(r+1) D \\
m
\end{array}\right) p^{m}(1-p)^{n-(r+1) D-m}\left(1-\frac{m D}{n-(r+1) D}\right) .
$$

Then, using the above equation in Equation (19), we have

$$
\begin{aligned}
& Q_{k}^{(j)}(n) \\
& =\left(\begin{array}{c}
n \\
k+\left\lfloor\frac{n}{D}\right\rfloor-j
\end{array}\right) p^{k+\left\lfloor\frac{n}{D}\right\rfloor-j}(1-p)^{n-\left(k+\left\lfloor\frac{n}{D}\right\rfloor-j\right)} \\
& \left.\quad+\sum_{r=0}^{\left\lfloor\frac{n}{D}\right\rfloor-j-1\left\lfloor\frac{n}{D}\right\rfloor-j-1-r} \sum_{m=0}^{n-(r+1) D} \begin{array}{c}
n-0 \\
m
\end{array}\right)\left(\begin{array}{c}
(r+1) D \\
k+r
\end{array}\right)\left(1-\frac{m}{\frac{n}{D}-r-1}\right) p^{m+k+r}(1-p)^{n-(m+k+r)} \\
& \quad-\sum_{r=0}^{\left\lfloor\frac{n}{D}\right\rfloor-j-1\left\lfloor\frac{n}{D}\right\rfloor-j-1-r} \sum_{m=0}^{n-\left(\begin{array}{c}
r+1) D \\
m
\end{array}\right)\left(\begin{array}{c}
(r+1) D \\
k+r+1
\end{array}\right)\left(1-\frac{m}{\frac{n}{D}-r-1}\right) p^{m+k+r+1}(1-p)^{n-(m+k+r+1)}}
\end{aligned}
$$


We then have the following simplified form:

$$
\begin{aligned}
& Q_{k}^{(j)}(n)=\left(\begin{array}{c}
n \\
k+\left\lfloor\frac{n}{D}\right\rfloor-j
\end{array}\right) p^{k+\left\lfloor\frac{n}{D}\right\rfloor-j}(1-p)^{n-\left(k+\left\lfloor\frac{n}{D}\right\rfloor-j\right)} \\
& \left.+\sum_{r=0}^{\left\lfloor\frac{n}{D}\right\rfloor-j-1\left\lfloor\frac{n}{D}\right\rfloor-j-1-r} \sum_{m=0}^{n-(r+1) D} \begin{array}{c}
n \\
m
\end{array}\right) p^{m}(1-p)^{n-(r+1) D-m} \\
& \times\left(\begin{array}{c}
(r+1) D \\
k+r
\end{array}\right) p^{k+r}(1-p)^{(r+1) D-(k+r)}\left(1-\frac{m}{\frac{n}{D}-r-1}\right) \\
& -\sum_{r=0}^{\left\lfloor\frac{n}{D}\right\rfloor-j-1\left\lfloor\frac{n}{D}\right\rfloor-j-1-r} \sum_{m=0}^{n-(r+1) D}\left(\begin{array}{c}
m \\
m
\end{array}\right) p^{m}(1-p)^{n-(r+1) D-m} \\
& \times\left(\begin{array}{c}
(r+1) D \\
k+r
\end{array}\right) p^{k+r}(1-p)^{(r+1) D-(k+r)}\left(1-\frac{m}{\frac{n}{D}-r-1}\right) \frac{p[(r+1) D-(k+r)]}{(1-p)(k+r+1)} .
\end{aligned}
$$

Using algebra, we can now obtain Equation (10) to complete the proof.

\section{Numerical Examples}

In this section, we show the numerical results. We use Equations (1) and (10) to compute the transient probabilities $Q_{0}^{(2)}(n), Q_{1}^{(2)}(n), Q_{2}^{(2)}(n)$ and $Q_{3}^{(2)}(n)$. Figure 1 shows the computation results of the probabilities.

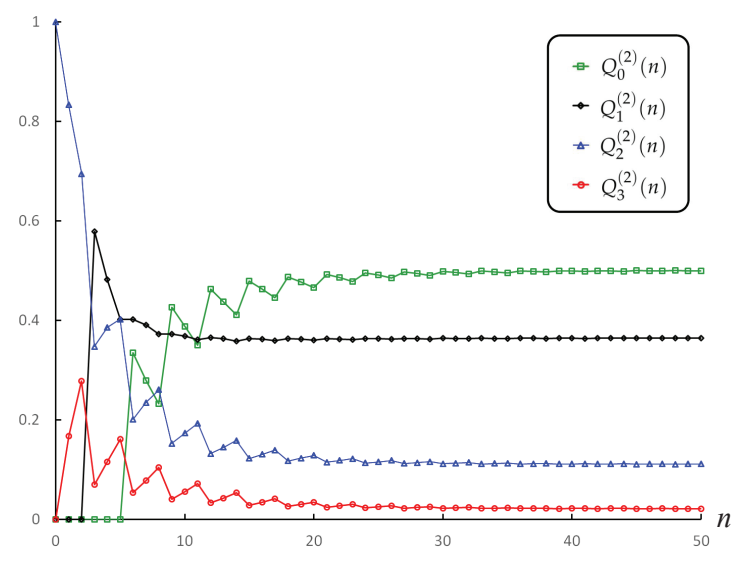

(a) $p=1 / 6, D=3,(\rho=p D=1 / 2)$

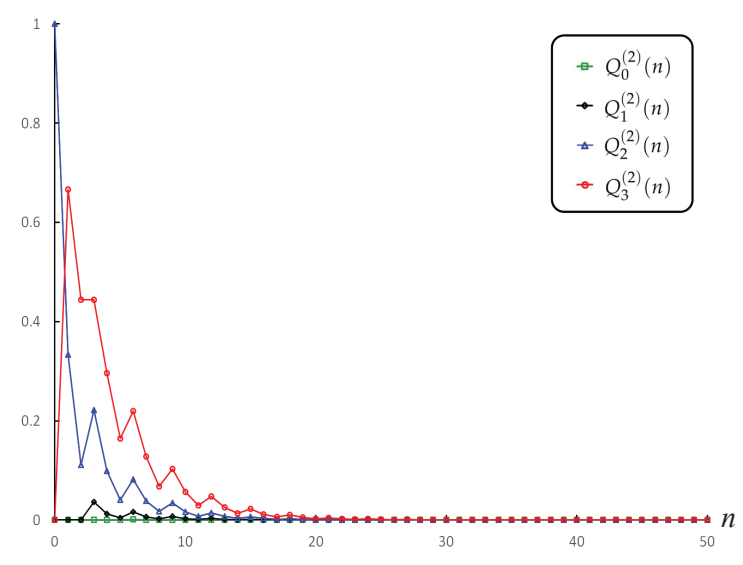

(b) $p=4 / 6, D=3,(\rho=p D=2)$

Figure 1. Probabilities $Q_{0}^{(2)}(n), Q_{1}^{(2)}(n), Q_{2}^{(2)}(n)$ and $Q_{3}^{(2)}(n)$.

Figure 1a shows the transient probabilities under the condition that $p=1 / 6$ and $D=3$ (i.e., $\rho=0.5)$. Let $P_{n}$ be the stationary probability of the Geo $/ D / 1$ queue. If $\rho<1$, we can use the result in Gravey et al. [19] to compute the stationary probability and obtain $P_{0}=0.5, P_{1}=0.364, P_{2}=0.11059$, and $P_{3}=0.02107$. Then, in Figure 1a, we can confirm that each transient queue-length probability converges to the stationary value. Figure $1 \mathrm{~b}$ shows the transient probabilities under the condition that $p=4 / 6$ and $D=3$ (i.e., $\rho=2$ ). When $\rho>1$, all the probabilities should be 0 as $n$ increases; we can confirm this in Figure 1b.

Author Contributions: Conceptualization: Y.H.B. and J.W.B.; Formal analysis: Y.H.B. and J.W.B.; Writing and original draft preparation: Y.H.B. and J.W.B.

Funding: The work of Y.H.B. was supported by the Basic Science Research Program through the National Research Foundation of Korea (NRF) funded by the Ministry of Education (NRF-2018R1D1A1B07043360). The work of J.W.B. was supported by the Basic Science Research Program through the National Research Foundation of Korea (NRF) funded by the Ministry of Education (NRF-2018R1D1A1B07043146). 
Conflicts of Interest: The authors declare no conflict of interest.

\section{References}

1. Griffiths, G.D.; Leonenko, G.M.; Williams, J.E. Time-dependent analysis of non-Empty $M / E_{k} / 1$ queue. Qual. Technol. Quant. Manag. 2008, 5, 309-320. [CrossRef]

2. Luchak, G. The continuous time solution of the equations of the single channel queue with a general class of service-time distributions by the method of generating functions. J. R. Stat. Soc. Ser. B (Methodol.) 1958, 20, 176-181. [CrossRef]

3. Saaty, L.T. Elements of Queueing Theory; Mcgraw Hill: New York, NY, USA, 1961.

4. Al-Seedy, R.O.; El-Sherbiny, A.A.; El-Shehawy, S.A.; Ammar, S.I. Transient solution of the M/M/c queue with balking and reneging. Comput. Math. Appl. 2009, 57, 1280-1285. [CrossRef]

5. Cheon, G.-S.; Choi, B.D.; Jin, S.-T. An application of Riordan arrays to the transient analysis of $M / M / 1$ queues. Appl. Math. Comput. 2014, 237, 659-671. [CrossRef]

6. Kijima, M. The transient solution to a class of Markovian queues. Comput. Math. Appl. 1992, $24,17-24$. [CrossRef]

7. Sharma, O.P.; Gupta, U.C. Transient Behaviour of an M/M/1/N queue. Stoch. Process. Their Appl. 1982, 13, 327-331. [CrossRef]

8. Tarabia, A.M.K. A new formula for the transient behaviour of a non-empty $M / M / 1 / \infty$ queue. Appl. Math. Comput. 2002, 132, 1-10. [CrossRef]

9. Griffiths, G.D.; Leonenko, G.M.; Williams, J.E. The transient solution to $M / E_{k} / 1$ queue. Oper. Res. Lett. 2006, 34, 349-354. [CrossRef]

10. Leonenko, G.M. A new formula for the transient solution of the Erlang queueing Model. Stat. Probab. Lett. 2009, 79, 400-406. [CrossRef]

11. Baek, J.W.; Lee, H.W.; Moon, S.K. A time-dependent busy period queue length formula for the $M / E_{k} / 1$ queue. Stat. Probab. Lett. 2014, 87, 98-104. [CrossRef]

12. Kapodistria, S.; Phung-Duc, T.; Resing, J. Linear Birth/Immigration-Death Process with Binomial Catastrophes. Probab. Eng. Inf. Sci. 2016, 30, 79-111. [CrossRef]

13. Garcia, J.M.; Brun, O.; Gauchard, D. Transient analytical solution of $M / D / 1 / N$ queues. J. Appl. Probab. 2002, 39, 853-864. [CrossRef]

14. Franx, G.J. A simple solution for the M/D/c waiting time distribution. Oper. Res. Lett. 2001, 29, $221-229$. [CrossRef]

15. Baek, J.W.; Lee, H.W.; Ahn, S.; Bae, Y.H. Exact timedependent solutions for the M/D/1 queue. Oper. Res. Lett. 2016, 44, 692-695. [CrossRef]

16. Parthasarathy, P.R.; Sudhesh, R. Exact transient solution of a discrete time queue with state-dependent rates. Am. J. Math. Manag. Sci. 2006, 26, 253-276. [CrossRef]

17. Kim, J. Transient analysis of the Geo/Goe/1 queue. J. Chungcheong Math. Soc. 2008, 21, 385-393.

18. Takagi, H. Queueing Analysis: A Foundation of Performance Evaluation; North Holland: Amsterdam, The Netherlands, 1993

19. Gravey, A.; Louvion, J.-R.; Boyer, P. On the Geo/D/1 and Geo/D/1/n queues. Perform. Eval. 1990, 11, 117-125. [CrossRef]

(c) 2019 by the authors. Licensee MDPI, Basel, Switzerland. This article is an open access article distributed under the terms and conditions of the Creative Commons Attribution (CC BY) license (http:/ / creativecommons.org/licenses/by/4.0/). 